\title{
Situación actual de Candida auris en Colombia, 2021
}

\section{Current situation of Candida auris in Colombia, 2021}

\author{
Isabella Martinez-Parada ${ }^{1, a}$, Esteban Agudelo-Quintero ${ }^{1,2, b}$, Diego Gerardo Prado-Molina ${ }^{1,2, c}$, \\ Juan Santiago Serna-Trejos ${ }^{1,2,3, d}$ \\ ${ }^{1}$ Facultad de Ciencias de la Salud, Programa de Medicina, Universidad Libre. Cali, Colombia. \\ ${ }^{2}$ Facultad de Ciencias de la Salud, Programa de Maestría en Epidemiologia, Universidad Libre. Cali, Colombia. \\ ${ }^{3}$ Grupo Colombiano de Investigación en Epidemiologia y Servicios, Universidad Libre. Cali, Colombia \\ ${ }^{a}$ ORCID: https://orcid.org/0000-0002-2309-4543 \\ ${ }^{b}$ ORCID: https://orcid.org/0000-0002-8801-9982 \\ ${ }^{\circ}$ ORCID: https://orcid.org/0000-0002-5402-8856 \\ d ORCID: https://orcid.org/0000-0002-3140-8995
}

\section{Correspondencia:}

Juan Santiago Serna Trejos

juansantiagosernatrejos@gmail.com

Recibido: 27 de agosto 2021

Aprobado: 6 de septiembre 2021

Publicación en línea: 8 de septiembre 2021

Conflictos de interés: Los autores declaran no tener conflictos de interés.

Fuente de financiamiento: Autofinanciado

Citar como: Martinez-Parada I, Agudelo-Quintero E, Prado-Molina D, Serna-Trejos J. Situación actual de Candida auris en Colombia, 2021. An Fac med. 2021;82(3):242-3. DOI: https://doi.org/10.15381/anales. v82i3.21089
An Fac med. 2021;82(3):242-3. / DOI: https://doi.org/10.15381/anales.v82i3.21089

\section{Sr. Editor,}

Hemos leído con gran interés el trabajo publicado recientemente en su revista médica, titulado: "Primer reporte de Candida auris en Perú" (1) bajo autoría de Paucar-Miranda y colaborares. La infección por Candida auris supone un reto para el control de las infecciones asociadas a la atención en salud (IAAS), puesto que se ha demostrado su aparición con estancias hospitalarias en pacientes que se benefician de estancias en unidades de cuidado intensivo.

El primer aislamiento de Candida auris en Colombia se realizó en el año 2015, en un estudio publicado por Ceballos-Garzón y colaboradores de manera prospectiva, donde se realizó la identificación inicial del patógeno con VITEK, al igual que el estudio realizado por Paucar-Miranda, con posteriores cultivos en medios CHROM agar y con la tipificación final bajo el "gold-standard" (MALDI-TOF) mediante prueba de espectrometría (2). Como variables poblacionales consideradas dentro del estudio de Ceballos-Garzón, se obtuvo de los 17 casos reportados, que un $88,2 \%$ de las muestras obtenidas pertenecieron a pacientes con requerimiento de hospitalización de cuidado intensivo, 94,1\% de los pacientes fueron portadores de dispositivos médicos (catéter venoso central, catéter urinario, ventilación mecánica entre otros) ${ }^{(2)}$.

Los factores de riesgo encontrados en ambos estudios para desarrollar candidiasis por C. auris en Colombia y Perú fueron: uso de dispositivos médicos (catéter venoso central), ventilación mecánica, diabetes, entre otros ${ }^{(1,2)}$. Inicialmente los aislamientos obtenidos en Colombia fueron: C. haemulonii, C. famata, C. albicans o C. tropicalis; se obtuvo su tipificación según el método hospitalario disponible, con requerimiento de confirmación por espectrometría (MALDI-TOF) para confirmación de C. auris ${ }^{(2)}$

La candidemia reporta incidencias de 1,18 casos por cada 1000 ingresos hospitalarios para Latinoamérica. En Colombia se estima una incidencia de 1,96 casos por cada 1000 ingresos hospitalarios, constituyendo una de las mayores de la región según datos de la Red de Micosis Invasivas de América Latina ${ }^{(3)}$.

Es preocupante las dificultades que surgen en aspectos de tipificación micológica para $C$. auris, puesto que no se tiene buena cobertura y acceso en nuestro país para el método considerado como el "gold- standard" (MALDI-TOF) y por supuesto las tipificaciones sesgadas de especies similares a C.auris arrojadas por métodos convencionales como el VITEK. Aunque la utilización de VITEK no es negativa del todo, pues esta permite tipificar múltiples resistencias a ciertos antifúngicos ${ }^{(3)}$.

El primer reporte de $C$. auris en la ciudad de Cali, la cual es la ciudad donde residen los autores de la presente carta, fue descrito por Castro-L en 2019, en el cual reportaron un paciente de 37 años de edad, con patologías reumatológicas de base, sin síntomas de sepsis, realizándose toma de muestras de punta de catéter venoso central. Como era de esperarse, 
inicialmente se aisló una especie diferente (C. haemulonii) por sistema Phoenix, quien posteriormente tuvo que ser rectificada por MALDI-TOF ${ }^{(4)}$.

Según el boletín epidemiológico más reciente asociado a IAAS, en Colombia, para la semana epidemiologia 09 (28 de febrero al 06 de marzo de 2021), C. auris estuvo asociado con brotes epidemiológicos, reportando 16 casos para el año 2020. En el mismo año 2020, los brotes en unidades de cuidado intensivo reportaron un $45 \%$ de aumento en el país ${ }^{(5)}$.

La contingencia global generada por SARS-CoV-2 ha impactado significativamente en Colombia en su asociación con casos reportados de $C$. auris, esto explicado probablemente por el gran aumento de ingresos a unidades de cuidado intensivo y el consecuente incremento del uso de dispositivos médicos invasivos. Se reportó para el año 2020, 340 casos confirmados con C. auris, los cuales se presentaron en pacientes hospitalizados con infección por SARS-CoV-2. Lo anterior ha encendido las alarmas sanitarias en los tiempos actuales de pandemia, en el cual se invita a realizar actividades de vigilancia e investigación epidemiológica, esclarecimiento de diagnostico de laboratorio, medidas de prevención y control de infecciones nosocomiales y comunitarias ${ }^{(6,7)}$.

\section{REFERENCIAS BIBLIOGRÁFICAS}

1. Paucar-Miranda C, Sandoval-Ahumada R, LópezMartínez R, Terrel-Gutierrez L, Zurita-Macalapu S, Urcia-Ausejo $\mathrm{F}$, et al. Primer reporte de Candida auris en Perú. An Fac med. 2021;82(1):56-61. DOI: https://doi.org/10.15381/anales.v82i1.20739

2. Morales-López SE, Parra-Giraldo CM, Ceballos-Garzón A, Martínez HP, Rodríguez GJ, Álvarez-Moreno $\mathrm{CA}$, et al. Invasive infections with multidrug-resistant yeast Candida auris, Colombia. Emerg Infect Dis 2017;23(1):162-4. DOI: 10.3201/eid2301.161497

3. Parra-Giraldo CM, Valderrama SL, Cortes-Fraile G, Garzón JR, Ariza BE, Morio F, et al. First report of sporadic cases of Candida auris in Colombia Int J Infect Dis. 2018;69:63-7. DOI: 10.1016/j. ijid.2018.01.034

4. Castro LÁ, Álvarez MI, Rojas F, Giusiano G, Martinez E. Candida auris infection in the central catheter of a patient without sepsis symptoms. Colomb Med. 2019;50(4):293-8. DOI: 10.25100/cm.v50i4.4248

5. Instituto Nacional de Salud de Colombia [Internet]. Boletin epidemiologico Semana Epidemiológica 09 28 de feb. al 06 de marzo de 2021. Minist Salud. 2021;9(1):67-67 [Fecha de acceso: 8 de agosto 2021]. Disponible en: https://www.ins.gov.co/ buscador-eventos/BoletinEpidemiologico/2021_Boletin_epidemiologico_semana_9.pdf

6. OPS/OMS [Internet]. Alerta Epidemiológica: Brotes de Candida auris en servicios de atención a la salud en el contexto de la pandemia de COVID-19. Organ Panam la Salud. 2021;1(1):1-11 [Fecha de acceso: 8 de agosto 2021]. Disponible en: https://iris.paho. org/bitstream/handle/10665.2/53377/EpiUpdate6February2021_spa.pdf?sequence=2\&isAllowed =y

7. Escandón P, Duarte C, Grupo de Microbiología - INS. Sandra Rivera EIA a la A en S- I. Alerta por emergencia global de infecciones invasivas causadas por la levadura multirresistente, Candida auris. Joruna hramacy [Internet]. 2017;(2):1-5. Disponible en: http://www.ins.gov.co/tramites-yservicios/examenes-de-interés-en-salud-publica/ Microbiologa/Alerta de C. auris.pdf 